\title{
State-space Coil Modelling in Plasma Magnetic Confinement Devices
}

\author{
Aitor J. Garrido ${ }^{1, *}$, Izaskun Garrido ${ }^{1}$, Diego González ${ }^{1}$, Odei Molinuevo ${ }^{1}$, Jon $_{\text {Lekube }}{ }^{\text {and }}$ Edorta Carrascal $^{2}$ \\ ${ }^{1}$ Automatic Control Group - ACG, Inst. of Research and Development of Processes - IIDP. Dept. Aut. Control and Systems \\ Eng., Faculty of Engineering of Bilbao, University of the Basque Country (UPV/EHU), $\mathrm{P}^{0}$ Rafael Moreno Moreno 3, Bilbao, \\ 48013. Spain \\ ${ }^{2}$ Automatic Control Group, Department of Thermal Engineering, Faculty of Engineering of Bilbao, University of the Basque \\ Country (UPV/EHU), 48013 Bilbao, Spain
}

\begin{abstract}
The need of robust and optimal control schemes is a key factor for the development of future fusion reactors. This paper has dealt with the state-space modelling of the Ultra-Low Iota Super Elongated Stellarator of the UPV/EHU, using a physical lumped parameter equivalent circuit approach. The model obtained has been validated by means of experimental output data showing an excellent matching with the real system. Besides, it has been designed a MPC scheme that has been successfully implemented both in simulation and experimentally using a real-time control platform.
\end{abstract}

\section{Introduction}

In the last years, it has been a considerable international effort to develop a clean technology based on magnetic confinement fusion energy, as it is the case of the ITER (International Thermonuclear Experimental Reactor) $[1,2]$. The process involves a nuclear reaction in which two cores of light atoms, usually hydrogen and its isotopes come together to form a heavier core. This reaction releases a great amount of energy in form of gamma rays and kinetic energy of the emitted particles that allows matter entering in state of plasma.

The final objective is to reproduce the process taking place in the stars, including the Sun, that constantly undergo nuclear fusion reactions. This represents a number of scientific and technical challenges. In particular, the automatic control composes one of the areas of interest regarding the development of a clean fusion technology, since one of the main limiting phenomena is the premature reaction decay due to instabilities of the plasma $[3,4]$.

Nowadays there exist two main magnetic fusion devices topologies: tokamaks and stellarators. On one hand tokamak reactors confine the plasma particles in a toroidal region using magnetic fields. These particles are held in small gyrating orbits by the magnetic field.
The principal magnetic field is usually toroidal and the confinement of the plasma is mainly achieved through the poloidal magnetic field. The field lines around the plasma current are combined with the toroidal field to produce helical field lines, which wrap around the torus in both directions. On the other hand, stellarators consist basically of a helically symmetric system bent into a torus with a large axisymmetric toroidal field, a moderately sized helical field and a small axisymmetric vertical field, all of them created by electromagnetic coils. In this way, the magnetic confinement in stellarators is fully achieved by the coils, so that no plasma current is needed to confine -although ohmic, non-inductive and bootstrap currents may also be present-. This fact makes stellarators a great alternative to tokamaks, with relevant progresses in the study of the physics of magnetically confined plasmas on these devices in the last years. Besides, its ability to operate in continuous mode -tokamaks are inherently pulsed devices- would allow a higher commercial profitability [5].

The studies presented in this paper have been performed for the UPV/EHU Stellarator ULISES (Ultra-Low Iota Super Elongated Stellarator), fully designed and constructed by the Automatic and Control Group of the UPV/EHU in collaboration with the EURATOM-CIEMAT (see Figure 1). 


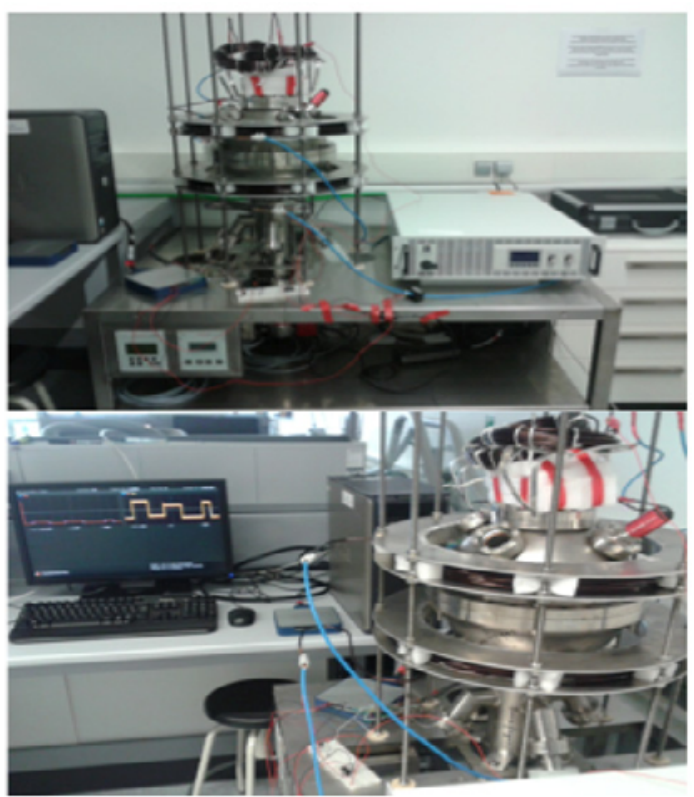

Fig. 1. UPV/EHU Stellarator ULISES

The rest of the paper is organized as follows: The model development is stated in Section 2. Section 2.1 describes the physical state-space model of the system considered, obtaining some relevant unknown parameter values by means of parameter identification over the real system. Then, the numerical solution achieved is benchmarking with experimental output data in Section 2.2, so as to validate the model. Section 3 is devoted to present the Model Predictive Controller (MPC) scheme used, both parameter description Section 3.1- and design and implementation of the real time control system -Section 3.2- employed to test the controller in the next section. Section 4 presents the corresponding result, showing the correspondence between the responses of the real system and the numerical model obtained. Finally, some concluding remarks are stated in the last section.

\section{Model statement}

In this section, the analytical development of the plant model is provided. For this purpose, the circuit formed by the coils and resistors composing the system plant is analysed so as to derive an adequate state-space representation. Afterwards, the model is validated by comparing the numerical plant output with the real system for different shots. It is shown that the output of the numerical model presents and excellent matching with that of the real plant for all them.

\subsection{System modelling}

Several studies on the behaviour of the device have been carried out in order to achieve an adequate equivalent system mode [6]. It has been determined that the internal circuit of the stellarator can be represented by the following lumped parameter electrical system (Figure 2). This lumped parameter approach is widely employed when in fusion devices [7-9]:

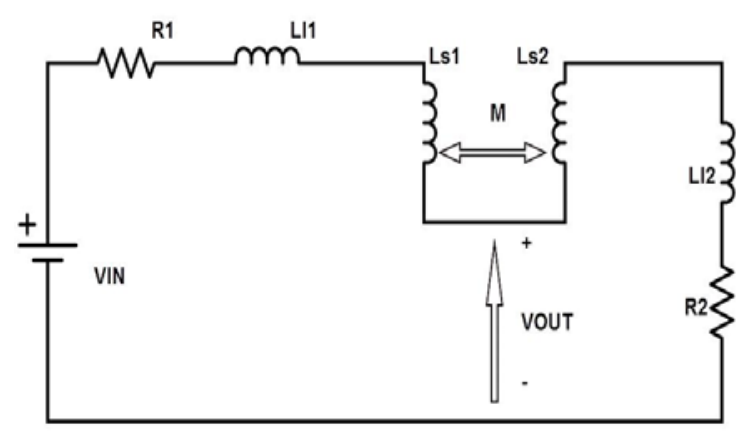

Fig. 2. Lumped parameter equivalent circuit

In this figure, $\mathrm{R}_{1}$ represents the total resistance of the first large coil and the first small inner coil. Analogously, $\mathrm{R}_{2}$ is the total resistance for the other two coils, so $\mathrm{R}$ represents the total resistance of the circuit. Note also that the inner small coils are coupled, so that total inductance of the circuit may be expressed as:

$K=L_{l 1}+L_{s 1}+L_{l 2}+L_{s 1} \pm M$,

with $\mathrm{M}$ being the mutual inductance of the two coupled coils and $\mathrm{K}$ the total inductance. The voltage input $\left(\mathrm{V}_{\mathrm{IN}}\right)$ represents the DC voltage supply and the voltage output is due to the drop in the second part of coils, so that:

$V_{\text {OUT }}=V_{R 2}+V_{L s 2}+V_{l 2}$.

Thus, the two basic equations which govern the circuit can be written as:

$$
\begin{aligned}
& V_{I N}=I\left(R_{1}+R_{2}\right)+K \frac{d I}{d t} \\
& V_{\text {OUT }}=I \cdot R_{2}+\left(L_{S 2}+L_{l 2}\right) \frac{d I}{d t}
\end{aligned}
$$

where I represents the current in the circuit. In order to obtain a state-space representation, the following state variables are chosen:

$x_{1}=I$

$x_{2}=\dot{x_{1}}=\frac{d I}{d t}$

Then, from equations (3) and (5), the following expression is achieved:

$\dot{x_{1}}=\frac{d I}{d t}=-\frac{\left(R_{1}+R_{2}\right)}{K} I+\frac{1}{K} V_{I N}$

Besides, deriving the equation (7) the result is:

$\dot{x_{2}}=\frac{d^{2} I}{d t^{2}}=-\frac{\left(R_{1}+R_{2}\right)}{K} \frac{d I}{d t}=-\frac{\left(R_{1}+R_{2}\right)}{K} x_{2}$

Therefore, substituting equations (5) in equations (7) and then (8), the state-space model of the system can 
be stablished in a convenient form with non-coupled state variables as follows:

$$
\begin{aligned}
& {\left[\begin{array}{l}
\dot{x_{1}} \\
\dot{x_{2}}
\end{array}\right]=\left[\begin{array}{cc}
-\frac{\left(R_{1}+R_{2}\right)}{K} & 0 \\
0 & -\frac{\left(R_{1}+R_{2}\right)}{K}
\end{array}\right]\left[\begin{array}{l}
x_{1} \\
x_{2}
\end{array}\right]+\left[\begin{array}{l}
\frac{1}{K} \\
0
\end{array}\right] V_{I N}} \\
& {\left[\begin{array}{l}
I_{\text {OUT }} \\
V_{\text {OUT }}
\end{array}\right]=\left[\begin{array}{cc}
1 & 0 \\
R_{2} & L_{S 2}+L_{l 2}
\end{array}\right]\left[\begin{array}{l}
x_{1} \\
x_{2}
\end{array}\right]}
\end{aligned}
$$

Now, is necessary to determine the real values of the parameters of the real system. For this purpose, some experimental tests are performed, collecting the results through the data acquisition system. In order to identify the elements of the matrices it is necessary to manage the differential equations of the system, so that using equations (7) and (8) it is possible to obtain an analytical expression for $x_{1}(t)$ :

$$
\begin{aligned}
x_{1}(t)=I(t)=\frac{V_{I N}}{R} & (1 \\
& \left.-e^{-\frac{R}{K} t}\right)
\end{aligned}
$$

Analogously, from (6) one has:

$$
\begin{aligned}
& x_{2}(t)=\frac{d I}{d t} \\
& =\frac{V_{I N}}{K} e^{-\frac{R}{K} t}
\end{aligned}
$$

Taking into account these results, it is possible to use a set of real data from appropriate experiments to calculate the parameters of the analytical solutions of $x_{1}(t)$ and $x_{2}(t)$ obtained. In this case, a typical step voltage signal with a $6 \mathrm{~V}$ magnitude has been used as input to the system. Considering a fixed instant during the transient response $-0.05 \mathrm{~s}$ from the enforcement of the step voltage- it is obtained a voltage output value of $2.47 \mathrm{~V}$ and a current value of $10.94 \mathrm{~A}$. Besides, the steady-state current value is $25.73 \mathrm{~A}$, so that the total resistance of the system can be directly stablished from Ohm's law providing value of $0.2332 \Omega$.

On the one hand, it is now possible from these data to obtain the total inductance of the circuit from equation (11), resulting in a value $K=0.021$. In the same way, from the solution (12) and the value of $K$ obtained, it is possible to calculate value of the current derivative of current at this specific point of the transient regime, resulting in $163.98 \mathrm{~A} / \mathrm{s}$. On the other hand, due to the symmetry of the stellarator, both large and small inner coil pairs present the same resistance, so that the value of is also known $-\mathrm{R}_{1}=\mathrm{R}_{2}=0.1166 \Omega$-. Finally, from these results and equation (4) it can also be calculated the value of $\mathrm{L}_{\mathrm{s} 2}+\mathrm{L}_{12}-7.28 \mathrm{mH}-$.

According to these results, the state-space model at this point may be expressed as:

$\left[\begin{array}{l}\dot{x_{1}} \\ \dot{x_{2}}\end{array}\right]=\left[\begin{array}{cc}-11.1 & 0 \\ 0 & -11.1\end{array}\right]\left[\begin{array}{l}x_{1} \\ x_{2}\end{array}\right]+\left[\begin{array}{c}47.62 \\ 0\end{array}\right] V_{I N}$
$\left[\begin{array}{l}I_{\text {OUT }} \\ V_{\text {OUT }}\end{array}\right]=\left[\begin{array}{cc}1 & 0 \\ 0.1166 & 0.00728\end{array}\right]\left[\begin{array}{l}x_{1} \\ x_{2}\end{array}\right]$

It has to be taken into account that the previous process has been performed for a particular state of the system during its transient response. In order to obtain a more accurate space-state model it has been used a grey-box modelling procedure, where the information acquired from the theoretical model determines the form of the identified system and the estimation of the elements of the state-space matrices is based on real input-output experimental data using the values previously obtained as initial values for the system identification algorithms [10]. In this way, it is possible to stablish a modelbased grey-box state-space representing the system:

$\left[\begin{array}{l}\dot{x_{1}} \\ \dot{x_{2}}\end{array}\right]=\left[\begin{array}{cc}-15.5 & 0 \\ 0 & -15.5\end{array}\right]\left[\begin{array}{l}x_{1} \\ x_{2}\end{array}\right]+\left[\begin{array}{c}69.3 \\ 0\end{array}\right] V_{I N}$
$\left[\begin{array}{l}I_{\text {OUT }} \\ V_{\text {OUT }}\end{array}\right]=\left[\begin{array}{cc}1 & 0 \\ 0.111 & 0.00632\end{array}\right]\left[\begin{array}{l}x_{1} \\ x_{2}\end{array}\right]$

As a result, the values of the Lumped parameter equivalent circuit physical circuit are: $\mathrm{K}=0.0144 \mathrm{H}$, $\mathrm{R}=0.2236 \Omega, \mathrm{R}_{2}=0.111 \Omega$ and $\mathrm{L}_{\mathrm{s} 2}+\mathrm{L}_{12}=6.32 \mathrm{mH}$.

\subsection{Model validation}

In this section the obtained model is compared with the real system output. For this purpose it has been used a set of validation data obtained for the same open-loop high demanding step-wise voltage input response. Results are shown in the following figures 3 and 4 both for voltage and current output, respectively. As it may be observed, the response of the grey-box model matches that of the real system, with a fit percentage of $74.2 \%$ for voltage output and $84.3 \%$ for current output case, being in part the error due to the noisy unfiltered experimental output.

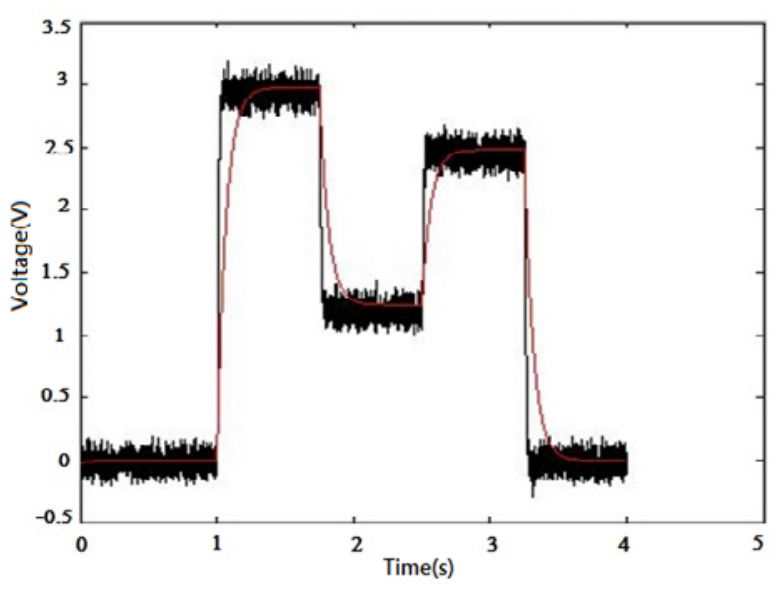

Fig. 3. Model vs real system open-loop responses ( $\left.\mathbf{V}_{\text {oUT }}\right)$ 


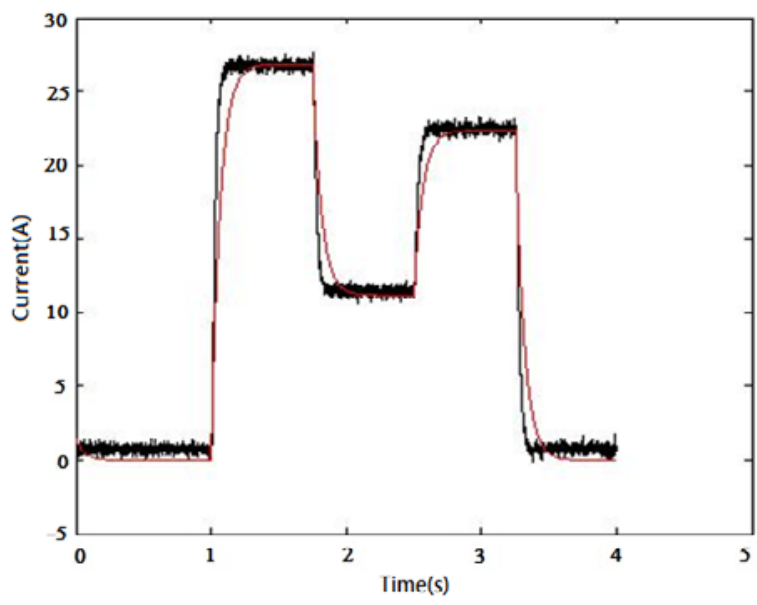

Fig. 4. Model vs real system open-loop responses ( $\left.\boldsymbol{I}_{\text {ovT }}\right)$

\section{Model predictive control law}

\subsection{Controller description}

The control objective is to regulate the voltage input supply to the stellarator to successfully solve the trajectory tracking problem for the voltage reference to be achieved in the chamber coils. In turn, current is controlled indirectly through voltage, as these signals are dependent each other. There exist different control approaches for this purpose, being the most used traditional PID-based schemes, robust (sliding-mode) schemes and MPC schemes [11-23]. In particular, when dealing with fusion devices where the available energy is limited, MPC is especially interesting due to its ability to ensure that both the control action and the solution error are minimal [9].

The working principle of MPC (Model Predictive Control) is to predict the future evolution of the outputs or manipulated variables [24-26]. For this purpose it is necessary to define a prediction horizon $N$ representing the time interval in which the output predictions evolve, so that the following sequence of output predictions are calculated according with system model considered and the information of evolution of the process until time $t$ :

$\hat{y}\left(t+\frac{1}{t}\right), \hat{y}\left(t+\frac{2}{t}\right), \ldots, \hat{y}\left(t+\frac{N}{t}\right)$

Besides, a control horizon $N_{u}$ representing the sequence of future control signals within $N$ is employed:

$u\left(\frac{t}{t}\right), u\left(t+\frac{1}{t}\right), \ldots, u\left(t+N-\frac{1}{t}\right)$

Usually, it is considered that the control signal remains constant for future time exceeding the control horizon $\left(N>N_{u}\right)$.

A further feature is the sliding horizon representing the number of components of the sequence of control signals vector $\boldsymbol{u}$ to be considered, neglecting the rest of the sequence at each iteration. The MPC scheme used is shown in Figure 5.

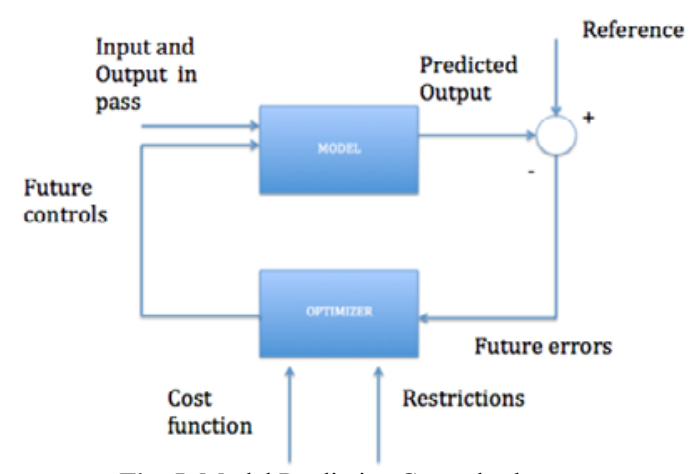

Fig. 5. Model Predictive Control scheme

The model used for the design of the MPC implementation is based on the previously obtained state-space (15-16) [27,28]. In order to develop an optimal controller, and given that the state variables are not coupled, the state-space model may be expressed as follows:

$\dot{x_{1}}=-15.5 x_{1}+69.3 V_{I N}$

$V_{\text {OUT }}=0.111 x_{1}$

The control interval of the controller is stablished in accordance with the experimental sample time of the real system $(0.00025 \mathrm{~s})$. The prediction horizon considered is set to 25 and the control horizon used is set to 13 (See Table 1). With these MPC parameters, the weight rate was 0.0225 and the weight of the output variable was stablished at $4[29,30]$.

Table 1. MPC parameters.

\begin{tabular}{|l|l|}
\hline Control Interval & $0,00025 \mathrm{~s}$ \\
\hline $\begin{array}{l}\text { Prediction Horizon } \\
\text { (Intervals) }\end{array}$ & 25 \\
\hline $\begin{array}{l}\text { Control Horizon } \\
\text { (Intervals) }\end{array}$ & 13 \\
\hline
\end{tabular}

\subsection{Real-time control system}

In order to demonstrate the feasibility and performance of the control scheme previously proposed, it has been implemented a real-time control design for the UPV/EHU Stellarator. The system has been designed using the real-time environment shown in Figure 6. The real-time applications are programmed and modified on an engineering PC and then launched to run on a dedicated real-time target computer connected to the physical system [31-32]. In particular, the control strategy is aimed to control a $15 \mathrm{~kW}$ EA-PSI 8080-510 3U power supply. A NI PCIe-6323 data acquisition device with two terminal blocks NI SCB$68 \mathrm{~A}$ has been used for the communication between the real-time control computer and the power source, the external sensors and the stellarator. The external sensors include a Hall effect inductive current sensor able to measure up to $600 \mathrm{~A}$ with an $1 \%$ error for measuring the current in the four-coil system of the stellarator and a scalable voltage sensor for measuring the output voltage. 


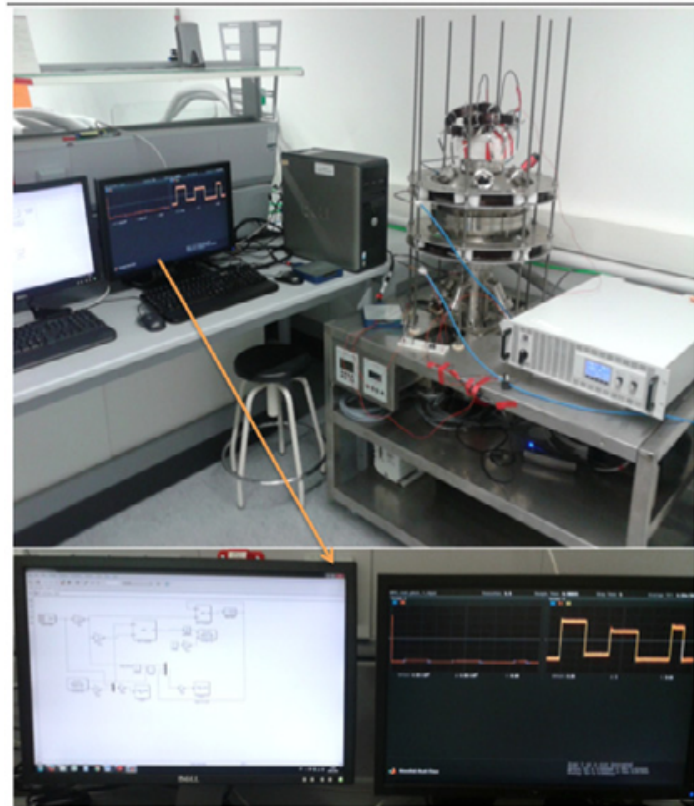

Fig. 6. Real-time control system

This structure allows to use the kernel of the real-time target computer only to run the real-time process, with an effective sample time below $250 \mu$ s, updating the data in the engineering $\mathrm{PC}$ at a lower rate via a RJ-45 Ethernet connection.

\section{Results: real system vs proposed model}

As it has been stated in the introduction the final aim is to develop different controllers for the UPV/EHU Stellarator. As a first step towards achieving this goal a grey-box model has been developed. This model has been initially validated against the real system. In a second step, a model predictive control (MPC) has been designed and implemented, both in simulation with the proposed model and experimentally. In order to validate the real-time MPC over the system, experimental voltage and current output results for a demanding shot may be seen, respectively, in Figure 7 and Figure 8.

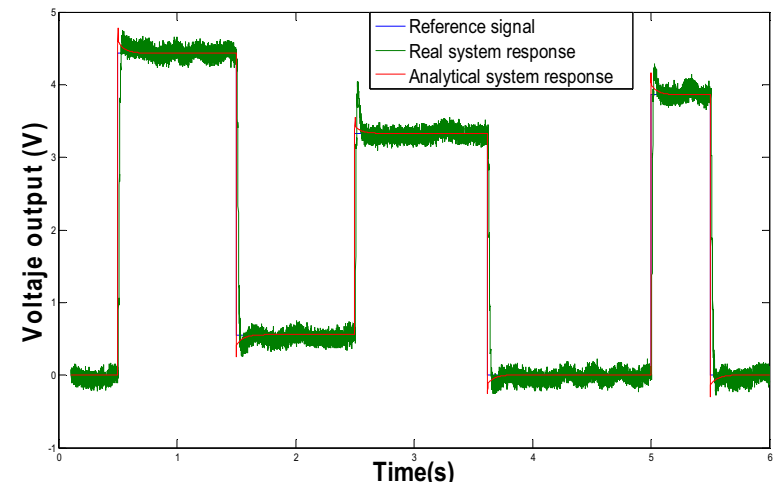

Fig. 7. Comparison of the responses of the real system and model for voltage output

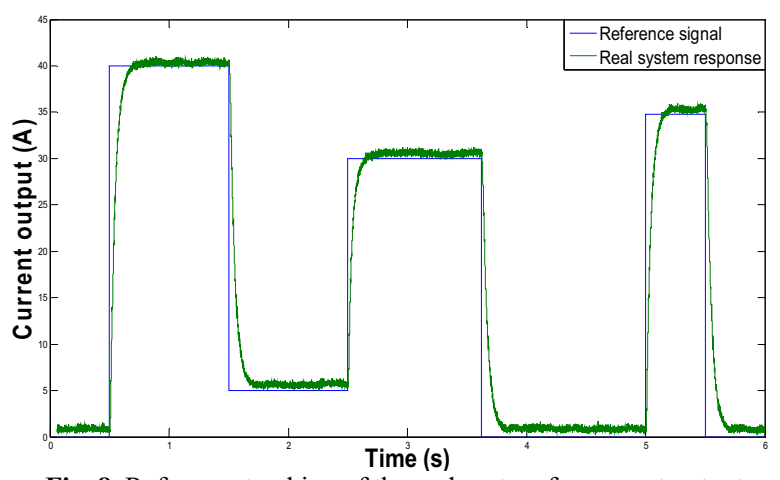

Fig. 8. Reference tracking of the real system for current output

As it may be observed, the controlled response of the real system is very similar to the desired one for both voltage and current, which shows that the real time MPC of the system provides an adequate performance over time, since the only deviation is a non-significant peak.

Besides, taking into account that the voltage is sufficiently controlled with the MPC and that the current depends basically on the voltage and on the resistance of the system, it can be assumed that this error is a result of a variation of the resistance of the system as the experiment evolves. This variation of the resistance may be due to the change of the temperature in the coils of the stellarator. Likewise, other factor that affects to the error is the environmental noise inherent to the experimental output, and in particular to the inductive current sensor of the coils.

Therefore, the real-time MPC has been proven to be an excellent candidate as a control scheme since it is able to optimize the future behaviour of the states while intrinsically minimizing the control action. In particular, Figure 7 and Figure 8 show how this scheme evidences the optimal prediction effect at each step in the reference value, where the overshoot is minimized, due to the control ability to predict the future flat-top regime.

\section{Conclusions}

This paper deals with the grey-box modelling of the magnetic confinement coil system of the UPV/EHU Stellarator. The state-space model obtained has been successfully validated by comparing the results of the model with those of the real system. Besides, a Model Predictive Control law has been experimentally implemented over the real-time control system using the proposed state-space model showing an excellent reference trajectory tracking behaviour. A benchmarking of the proposed MPC with a traditionally used PID controller has been also performed. It has been shown that the MPC presents a superior performance due to its ability to predict the state of the plant during the dynamic operation, while PID controller needs a fine tuning when dealing with time-delay or changing dynamics during operation. In particular, the proposed MPC scheme allows ensuring both minimal control action and solution error, which 
is especially relevant in the case of fusion devices where the available energy is limited.

This work was supported in part by the University of the Basque Country (UPV/EHU) through Project PPG17/33 and by the MINECO through the Research Project DPI201570075-R (MINECO/FEDER, UE).

The authors would also like to thank the collaboration of the Basque Energy Board (EVE) through Agreement UPV/EHUEVE23/6/2011, the Spanish National Fusion Laboratory (EURATOM-CIEMAT) through Agreement UPV/EHUCIEMAT08/190 and Stefano Coda and the TCV team for its collaboration and help.

\section{References}

1. ITER Web site. http://www.iter.org/ (2017)

2. Teller, E. (Ed.). Fusion Part A: Magnetic Confinement. Elsevier (2012)

3. Sevillano, M. G., Garrido, I., \& Garrido, A. J. "Control-oriented automatic system for transport analysis (ASTRA)-matlab integration for tokamaks". Energy 36 (5), 2812-2819. DOI: 10.1016/j.energy.2011.02.022 (2011)

4. Sevillano, M.G., Garrido, I., Garrido, A. J., Romero, J.A., Paley, J., Coda, S. and the TCV team "Observer-based real-time control for the poloidal beta of the plasma using diamagnetic measurements in tokamak fusion reactors", Proceedings of the IEEE Conference on Decision and Control, art. no. 6160260, pp. 7536-7542. DOI: 10.1109/CDC.2011.6160260 (2011)

5. Menard, J. E., Bromberg, L., Brown, T., Burgess, T., Dix, D., El-Guebaly et al. Prospects for pilot plants based on the tokamak, spherical tokamak and stellarator. Nuclear Fusion, 51(10), 103014 (2011)

6. Neubauer, O., Panin, A., Czymek, G., \& Giesen, B. Approaches to numerical modeling in development process of complex structures for fusion devices. In Fusion Engineering (SOFE), 2013 IEEE 25th Symposium on, pp. 1-8 (2013)

7. Romero, J.A., Moret, J. M., Coda, S., Felici, F., \& Garrido, I. "Development and validation of a tokamak skin effect transformer model", Nuclear Fusion, 52 (2), art. no. 023019 (2012)

8. Garrido, A.J., Garrido, I., Goretti Sevillano, M., Alberdi, M., Amundarain, M., Barambones, O., De La Sen, M. "Nuclear fusion control-oriented plasma current linear models" Proc. Of the International Conference on Systems 2014. Corfu. Greece, pp. 145-150 (2010)

9. Garrido I, Garrido A.J., Romero J.A., Carrascal E. Sevillano-Berasategui M. and Barambones O., "Low Effort LI Nuclear Fusion Plasma Control Using Model Predictive Control Laws," Mathematical Problems in Engineering, Article ID 527420. DOI: 10.1155/2015/527420 (2015)

10. Ljung 1.. "Some Classical and Some New Ideas for Identification of Linear Systems" Journal of
Control, Automation and Electrical Systems. Volume 24, Issue 1-2 (2013)

11. Garrido, I., Garrido, A.J., Sevillano, M.G., \& Romero, J.A., "Robust sliding mode control for tokamaks", Mathematical Problems in Engineering, 2012, art. no. 341405. DOI: 10.1155/2012/341405 (2012)

12. Garrido, A. J., Garrido, I., Amundarain, M., Alberdi, M., \& De La Sen, M. "Sliding-mode control of wave power generation plants". IEEE Transactions on Industry Applications, 48 (6), 2372-2381. DOI: 10.1109/TIA.2012.2227096 (2012)

13. Garrido, I., Garrido, A.J., Sevillano, M.G., Romero, J.A., Amundarain, M., \& Alberdi, M. "Tokamak state-space control modeling" Canadian Conference on Electrical and Computer Engineering, CCECE Vols. 1-4 0840-7789 (2008)

14. Alkorta, P., Barambones, O., Garrido, A.J., Garrido, I. "SVPWM variable structure control of induction motor drives" IEEE International Symposium on Industrial Electronics, art. no. 4374768, pp. 1195-1200 (2007)

15. Garrido, A.J., Garrido, I., Alberdi, M., Amundarain, M., Barambones, O., Romero, J.A. "Robust control of oscillating water column (OWC) devices: Power generation improvement" OCEANS 2013 MTS/IEEE - San Diego: An Ocean in Common, art. no. 6740982 (2013)

16. Garrido, I., Garrido, A.J., Alberdi, M., Amundarain, M., Barambones, O. "Performance of an ocean energy conversion system with DFIG sensorless control" Mathematical Problems in Engineering, 2013, art. no. 260514. DOI: 10.1155/2013/260514 (2013)

17. Garrido, A.J., De La Sen, M., Soto, J.C., Barambones, O., Garrido, I. "Suboptimal regulation of a class of bilinear interconnected systems with finite-time sliding planning horizons". Mathematical Problems in Engineering, 2008, art. no. 817063 . DOI: $10.1155 / 2008 / 817063$ (2008)

18. Sevillano, G., Garrido, I., \& Garrido, A. J. "ASTRA-matlab integration for the control of tokamaks" (2011). Informatica, 22 (2), 241-257. ISSN: 08684952

19. Garrido, A.J., Otaola, E., Garrido, I., Lekube, J., Liria, P., Mader, J., "OWC wave power plants capture chamber modelling" OCEANS 2015 MTS/IEEE Washington, art. no. 7401863. ISBN: 978-0-9339-5743-5 (2016)

20. Garrido, I., Garrido, A.J., Coda, S., Le, H.B., Moret, J.M., Real time hybrid model predictive control for the current profile of the tokamak à configuration variable (TCV). Energies, 2008, 9 (8), art. no. 609. DOI: 10.3390/en9080609 (2008)

21. Garrido, I., Garrido, A.J., Lekube, J., Otaola, E., Carrascal, E. "Oscillating water column control and monitoring". OCEANS 2016 MTS/IEEE 
Monterey CA, art. no. 7761420, DOI: 10.1109/OCEANS.2016.7761420 (2016)

22. Garrido, A.J., Garrido, I., Lekube, J., De La Sen, M., Carrascal, E. "Modeling of Oscillating Water Column wave energy systems". World Automation Congress, 2016-October, art. no. 7583008, DOI: 10.1109/WAC.2016.7583008 (2016)

23. Lekube, J., Garrido, A.J., Garrido, I., Rotational Speed Optimization in Oscillating Water Column Wave Power Plants Based on Maximum Power Point Tracking (2016) IEEE Transactions on Automation Science and Engineering, Article in Press. DOI: 10.1109/TASE.2016.2596579 (2016)

24. Cheng, Q., Cannon, M., \& Kouvaritakis, B. The design of dynamics in the prediction structure of robust MPC. International Journal of Control, 86(11), 2096-2103 (2013)

25. Morari, M., Garcia, C. E., \& Prett, D. M. Model predictive control: theory and practice. In Proc./FAC Workshop on Model Based Process Control (pp. 1-12). (2014)

26. Carrascal, E., Garrido, I., Garrido, A.J., Sala, J.M. "Model Predictive Control for the heating system of a public building". World Automation Congress Proceedings, art. no. 6935986, pp. 433-438. (2014)

27. Garrido, I., Romero, J.A., Garrido, A.J., Lucchin, D., Carrascal, E., Sevillano-Berasategui, G. "Internal inductance predictive control for Tokamaks". World Automation Congress Proceedings, art. no. 6936072, pp. 628-633. DOI: 10.1109/WAC.2014.6936072 (2014)

28. Garrido, A.J., Garrido, I., Barambones, O., Alkorta, P., Maseda, F.J. "Simple linear models for plasma control in Tokamak Reactors" 2008 International Conference on Control, Automation and Systems, ICCAS 2008, art. no. 4694261, pp. 2429-2432. DOI: 10.1109/ICCAS.2008.4694261 (2008)

29. Wang, L. Model predictive control system design and implementation using MATLABß. Springer Science \& Business (2009)

30. Veselý, V., Rosinová, D., \& Foltin, M. Robust model predictive control design with input constraints. ISA transactions, 49(1), 114-120. (2010)

31. Yin, S., Luo, H., \& Ding, S. X. Real-time implementation of fault-tolerant control systems with performance optimization. Industrial Electronics, IEEE Transactions on, 61(5), 24022411 (2014)

32. Buttazzo, G. C. Hard real-time computing systems: predictable scheduling algorithms and applications (Vol. 24). Springer Science \& Business Media (2011) 